Citation: BLAKE, H, BENNETT, E and BATT, ME, 2014. Evaluation of occupational health checks for hospital employees In: Proceedings of the 10th UK Society for Behavioural Medicine Annual Scientific Meeting, 3-4 December, Nottingham.

\title{
Evaluation of occupational health checks for hospital employees
}

Holly Blake ${ }^{1}$, Eleanor Bennett ${ }^{2}$, Mark. E. Batt ${ }^{0}$

${ }^{1}$ University of Nottingham, Nottingham, UK, ${ }^{2}$ Nottingham University Hospitals NHS Trust, Nottingham, UK

Aims: To ascertain which employees choose to access occupational health checks $(\mathrm{OHC}$ 's), their perceptions of the usefulness of information received and whether they choose to act on tailored advice provided.

Methods: 253 hospital employees attended workplace $\mathrm{OHC}$ then completed online questionnaire survey.

Results: Participants included new cases $(80 \%)$ and those who had accessed the service previously (20\%), all age categories (23-69 years) and all occupational groups, although the vast majority were in officebased sedentary job roles, nursing or allied health professions $(78.3 \%)$. Almost half were overweight or obese $(46.7 \%)$; many reported existing health problems or family history of chronic disease. Participants perceived occupational health checks to be convenient, informative and useful for raising their awareness of health issues, reassurance and monitoring, early identification of potential health problems and signposting to appropriate services. Participants reported post-check dietary changes $(41 \%)$ and increases in physical activity $(30 \%)$; smokers reported quitting or cutting down (44\%) and those exceeding alcohol limits reported cutting frequency or units of consumption (48\%). More than half those advised to visit their GP complied (53\%).

Conclusions: General health checks can be feasibly delivered in a multisite hospital workplace setting with diverse appeal. Provision of tailored health information can help to raise health awareness and motivate health behaviour change or maintenance amongst hospital employees, including those reporting risk factors for chronic disease. Employees value the investment of healthcare organisations in the health and wellbeing of their workforce. 